\title{
Anti-CD19 Gold Nanostars as New Therapeutic Vectors for the Treatment of Acute Lymphoblastic Leukemia
}

\author{
Tatar Andra-Sorina ${ }^{1,2}$, Nagy-Simon Timea ${ }^{1}$, Jurj Ancuta $^{3}$, Berindan-Neagoe Ioana ${ }^{3}$, Tomuleasa Ciprian ${ }^{3}$, \\ Cialla-May Dana ${ }^{4}$, Astilean Simion ${ }^{1,2}$, Boca Sanda ${ }^{1,}$ \\ ${ }^{1}$ Nanobiophotonics and Laser Microspectroscopy Center, Interdisciplinary Research Institute on Bio-Nano-Sciences \\ Babes-Bolyai University \\ T. Laurian 42, 400271 Cluj-Napoca, Romania \\ simion.astilean@phys.ubbcluj.ro, sanda.boca@phys.ubbcluj.ro \\ ${ }^{2}$ Faculty of Physics, Babes-Bolyai University \\ Kogalniceanu 1, 400084 Cluj-Napoca, Romania \\ ${ }^{3}$ Research Center for Functional Genomics and Translational Medicine \\ Iuliu Haţieganu University of Medicine and Pharmacy \\ Marinescu Street 23, 400337 Cluj-Napoca, Romania \\ ${ }^{4}$ Leibniz Institute of Photonic Technology Jena \\ Albert-Einstein-Strasse 9, 07745 Jena, Germany
}

\section{Extended Abstract}

Acute lymphoblastic leukemia (ALL) is the most frequent malignancy in children, and the second most common in adolescents. This disease is characterised by a wide palette of genetic causes, which create many possible protein expression profiles, allowing for a targeted treatment for each patient [1]. Lately, antibody-based nano-treatments were developed, aiming for a more patient-specific tailored approach, and thus better outcomes [2].

The objective of our work is to specifically target ALL cells using antibody-tagged nanoparticles and to demonstrate improved cell uptake, feature that will be later harnessed for further treatment options. Our scope is to perform a series of cellular tests, where cells are incubated with targeted and non-targeted nanoparticles, and to monitor the outcome of the experiment. Targeted nanoparticles are more likely to get in direct contact to the cell surface by specific antibody-antigen interactions, thus increasing the internalisation probability. Using methods such as dark-field microscopy imaging, confocal Raman imaging, and transmission electron microscopy, we monitor the presence and localisation of our nanoparticle complexes at the cellular level. Also, by employing cell toxicity, viability and proliferation assays we assess the therapeutic effect of the conjugated particles onto ALL cells.

In this study, we prepared star-shaped gold nanoparticles by a seed-mediated chemical synthesis, we biocompatilised the particles with PEG polymer and conjugated the particles with anti-CD19 antibody specifically selected for ALL cell targeting [3]. Further on, we marked the nanoconjugates with Nile Blue molecule, which can act as a Raman reporter. The formed nanocomplexes were thoroughly characterised by transmission electron microscopy, UV-Vis spectroscopy, dynamic light scattering and zeta-potential measurements. Various concentrations of nanocomplexes were incubated in the presence of B lymphoblast CCRF-SB cells for 24, 48, and 72 hours. Thus far, our study showed efficient nanoparticle internalisation, as seen in the obtained confocal Raman maps and dark field microscopy images. Cytotoxicity tests revealed little effect when particles are delivered in small concentrations, while statistically significant effects were observed for higher concentrations starting with the fourth day of administration. Further tests included the quantification of the level of particle internalisation for a more precise understanding of the intracellular effects caused by the nanocomplex, and more thorough analyses of their cytotoxic effect.

\section{Acknowledgements}

This work was supported by project PN-II-RU-TE-2014-4-2426 (NanoMEDLeuKemist). 


\section{References}

[1] A-S. Tatar, T. Nagy-Simon, C. Tomuleasa, S. Boca, and S. Astilean, "Nanomedicine approaches in acute lymphoblastic leukemia," J. Controlled Release, vol. 238, pp. 123-138, 2016.

[2] D. C. Julien, S. Behnke, G. Wang, G. K. Murdoch, and R. A. Hill, "Utilization of monoclonal antibody-targeted nanomaterials in the treatment of cancer," $m A b s$, vol. 3, no. 5, pp. 467-478, 2011.

[3] K. Laginha, D. Mumbengegwi, and T. Allen, "Liposomes targeted via two different antibodies: Assay, B-cell binding and cytotoxicity," Biochim. Biophys. Acta BBA - Biomembr., vol. 1711, no. 1, pp. 25-32, 2005. 